ternational Electronic Journal of Algebra

Volume 26 (2019) 87-94

DOI: $10.24330 /$ ieja.586962

\title{
ANNIHILATORS OF TOP LOCAL COHOMOLOGY MODULES DEFINED BY A PAIR OF IDEALS
}

\author{
S. Karimi and Sh. Payrovi \\ Received: 6 November 2018; Revised: 20 February 2019; Accepted: 24 February 2019 \\ Communicated by Burcu Üngör \\ Abstract. Let $R$ be a commutative Noetherian ring, $I, J$ two proper ideals of \\ $R$ and let $M$ be a non-zero finitely generated $R$-module with $c=\operatorname{cd}(I, J, M)$. \\ In this paper, we first introduce $T_{R}(I, J, M)$ as the largest submodule of \\ $M$ with the property that $\operatorname{cd}\left(I, J, T_{R}(I, J, M)\right)<c$ and we describe it in \\ terms of the reduced primary decomposition of zero submodule of $M$. It is \\ shown that $\operatorname{Ann}_{R}\left(H_{I, J}^{d}(M)\right)=\operatorname{Ann}_{R}\left(M / T_{R}(I, J, M)\right)$ and $\operatorname{Ann}_{R}\left(H_{I}^{d}(M)\right)=$ \\ $\operatorname{Ann}_{R}\left(H_{I, J}^{d}(M)\right)$, whenever $R$ is a local ring, $M$ has dimension $d$ with $H_{I, J}^{d}(M)$ \\ $\neq 0$ and $J^{t} M \subseteq T_{R}(I, M)$ for some positive integer $t$.
}

Mathematics Subject Classification (2010): 13D45, 14B15

Keywords: Attached prime ideal, top local cohomology module

\section{Introduction}

Local cohomology theory has been a significant tool in commutative algebra and algebraic geometry. As a generalization of the usual local cohomology modules, Takahashi, Yoshino and Yoshizawa [12] introduced the local cohomology modules with respect to a pair of ideals. To be more precise, suppose that $R$ is a commutative Noetherian ring and $I, J$ are two ideals of $R$. Let $W(I, J)=\left\{\mathfrak{p} \in \operatorname{Spec}(R): I^{n} \subseteq \mathfrak{p}+\right.$ $J$ for some positive integer $n\}$. For an $R$-module $M$, the $(I, J)$-torsion submodule $\Gamma_{I, J}(M)$ of $M$, which consists of all elements $x$ of $M$ with $\operatorname{Supp}(R x) \subseteq W(I, J)$, is considered. Let $i$ be an integer, the local cohomology functor $H_{I, J}^{i}$ with respect to $(I, J)$ is defined to be the $i$-th right derived functor of $\Gamma_{I, J}$. The $i$-th local cohomology module of $M$ with respect to $(I, J)$ is denoted by $H_{I, J}^{i}(M)$. When $J=0$, then $H_{I, J}^{i}$ coincides with the usual local cohomology functor $H_{I}^{i}$ with the support in the closed subset $V(I)$.

One of the basic problems concerning local cohomology modules is to determine the annihilators of them. This problem for ordinary local cohomology modules has been studied by several authors, see $[6,7,8,9,11]$, and has led to some interesting results. In particular, Bahmanpour et al. in [2] proved an interesting result about 
the annihilator of $H_{\mathfrak{m}}^{d}(M)$ the $d$-th local cohomology module of $M$, when $(R, \mathfrak{m})$ is a complete local ring and $M$ is a non-zero finitely generated $R$-module with $d=\operatorname{dim} M$. Then Atazadeh et. al. [1] generalized this fact to the local cohomology modules with respect to an arbitrary ideal $I$.

They first defined $T_{R}(I, M)$ as the largest submodule of $M$ such that $\operatorname{cd}\left(I, T_{R}(I, M)\right)$ $<c$, in which $c=\operatorname{cd}(I, M)$, see [1, Definition 2.2], and then they proved the following fact.

Theorem 1.1. [1, Theorem 2.3] Let $R$ be a Noetherian ring and $I$ be an ideal of $R$. Let $M$ be a non-zero finitely generated $R$-module of dimension d such that $H_{I}^{d}(M) \neq 0$. Then $\operatorname{Ann}_{R}\left(H_{I}^{d}(M)\right)=\operatorname{Ann}_{R}\left(M / T_{R}(I, M)\right)$.

The purpose of the present paper is to introduce $T_{R}(I, J, M)$ as the largest submodule of $M$ with the property that $\operatorname{cd}\left(I, J, T_{R}(I, J, M)\right)<c$, in which $c=$ $\operatorname{cd}(I, J, M)$. Next in Corollary 2.3 we relate this submodule of $M$ with another special submodules $T_{R}(I, M)$ and $T_{R}(\mathfrak{m}, M)$ of $M$. Then we describe in more detail the structure of $T_{R}(I, J, M)$ in terms of the reduced primary decomposition of the zero submodule of $M$ in Theorem 2.4. Namely, if $0=\cap_{i=1}^{n} N_{i}$ denotes a reduced primary decomposition of the zero submodule in $M$ such that $N_{i}$ is a $\mathfrak{p}_{i}$-primary submodule of $M$, for all $i=1, \cdots, n$, then

$$
T_{R}(I, J, M)=\cap_{\mathrm{cd}\left(I, J, R / \mathfrak{p}_{i}\right)=c} N_{i} .
$$

Pursuing this point of view further we establish some results about the annihilator of top local cohomology modules with respect to a pair of ideals $I, J$. More precisely, as a main result of this paper, we derive the following consequence.

Theorem 1.2. Let $R$ be a Noetherian local ring and $I, J$ be two ideals of $R$. Let $M$ be a non-zero finitely generated $R$-module with dimension d such that $H_{I, J}^{d}(M) \neq 0$. Then

(i) $\operatorname{Ann}_{R}\left(H_{I, J}^{d}(M)\right)=\operatorname{Ann}_{R}\left(M / T_{R}(I, J, M)\right)$.

(ii) If $J^{t} M \subseteq T_{R}(I, M)$ for some positive integer $t$, then $\operatorname{Ann}_{R}\left(H_{I}^{d}(M)\right)=$ $\operatorname{Ann}_{R}\left(H_{I, J}^{d}(M)\right)$.

For notations and terminologies not given in this paper, the reader is referred to [3] if necessary.

\section{Annihilators of local cohomology modules}

Throughout this section $R$ is a commutative Noetherian ring, $I, J$ are two proper ideals of $R$ and $M$ is a non-zero finitely generated $R$-module. Let $\operatorname{cd}(I, J, M)$ be 
the supremum of all integers $r$ for which $H_{I, J}^{r}(M) \neq 0$. We call this integer the cohomological dimension of $R$-module $M$ with respect to $I, J$. When $J=0$, we have that $\operatorname{cd}(I, 0, M)=\operatorname{cd}(I, M)$, which is just the supremum of all integers $r$ for which $H_{I}^{r}(M) \neq 0$. In [5, Corollary 3.3] a characterization for $\operatorname{cd}(I, J, M)$ is provided

$$
\operatorname{cd}(I, J, M)=\inf \left\{i \mid H_{I, J}^{i}(R / \mathfrak{p})=0 \text { for all } \mathfrak{p} \in \operatorname{Supp}_{R}(M)\right\}-1
$$

Lemma 2.1. [5, Proposition 3.2] Let $M$ and $N$ be two finitely generated $R$-modules such that $\operatorname{Supp}_{R} N \subseteq \operatorname{Supp}_{R} M$. Then $\operatorname{cd}(I, J, N) \leq \operatorname{cd}(I, J, M)$.

Definition 2.2. Let $R$ be a Noetherian ring and $I, J$ be two ideals of $R$. Let $M$ be a non-zero finitely generated $R$-module. We denote by $T_{R}(I, J, M)$ the largest submodule of $M$ such that $\operatorname{cd}\left(I, J, T_{R}(I, J, M)\right)<\operatorname{cd}(I, J, M)$. It is easy to see that $T_{R}(I, J, M)=\cup\{N: N \leq M$ and $\operatorname{cd}(I, J, N)<\operatorname{cd}(I, J, M)\}$. When $J=0$ this definition coincides with that of [1, Definition 2.2].

Corollary 2.3. Let $(R, \mathfrak{m})$ be a Noetherian local ring and $M$ be a non-zero finitely generated $R$-module of dimension d such that $H_{I, J}^{d}(M) \neq 0$. Then $T_{R}(\mathfrak{m}, M) \subseteq$ $T_{R}(I, M) \subseteq T_{R}(I, J, M)$.

Proof. For the first inclusion let $x \notin T_{R}(I, M)$. Then $\operatorname{cd}(I, R x)=d$ and so $H_{I}^{d}(R x) \neq 0$. Thus $\operatorname{dim} R x=d$ and by [3, Theorem 6.1.4], $H_{\mathfrak{m}}^{d}(R x) \neq 0$. Hence, $x \notin T_{R}(\mathfrak{m}, M)$ and therefore $T_{R}(\mathfrak{m}, M) \subseteq T_{R}(I, M)$. Now, let $x \notin T_{R}(I, J, M)$. Then $\operatorname{cd}(I, J, R x)=d$ so that $H_{I, J}^{d}(R x) \neq 0$. Now, by [4, Theorem 2.1] we have $\emptyset \neq \operatorname{Att}_{R}\left(H_{I, J}^{d}(R x)\right) \subseteq \operatorname{Att}_{R}\left(H_{I}^{d}(R x)\right)$. Hence, $H_{I}^{d}(R x) \neq 0$ and $\operatorname{cd}(I, R x)=d$. Therefore, $x \notin T_{R}(I, M)$ we have the desired result.

Theorem 2.4. Let $M$ be a non-zero finitely generated $R$-module with cohomological dimension $c=\operatorname{cd}(I, J, M)$. Then

$$
T_{R}(I, J, M)=\cap_{\mathrm{cd}\left(I, J, R / \mathfrak{p}_{i}\right)=c} N_{i} .
$$

Here, $0=\cap_{i=1}^{n} N_{i}$ denotes a reduced primary decomposition of the zero submodule of $M$ and $N_{i}$ is a $\mathfrak{p}_{i}$-primary submodule of $M$.

Proof. We first show that $T_{R}(I, J, M) \subseteq \cap_{\mathrm{cd}\left(I, J, R / \mathfrak{p}_{i}\right)=c} N_{i}$ and then we have the desired result whenever $\operatorname{cd}\left(I, J, \cap_{\mathrm{cd}\left(I, J, R / \mathfrak{p}_{i}\right)=c} N_{i}\right)<c$. Let $x \in T_{R}(I, J, M)$. Then $\operatorname{cd}(I, J, R x)<c$ and so $H_{I, J}^{c}(R x)=0$. Thus for each $\mathfrak{p} \in \operatorname{Ass}_{R}(R x)$ we have $H_{I, J}^{c}(R / \mathfrak{p})=0$. Hence, $\operatorname{Ass}_{R}(R x) \subseteq\left\{\mathfrak{p}_{i}: \mathfrak{p}_{i} \in \operatorname{Ass}_{R} M, \operatorname{cd}\left(I, J, R / \mathfrak{p}_{i}\right)<c\right\}$ and 
therefore,

$$
\bigcap_{\operatorname{cd}\left(I, J, R / \mathfrak{p}_{i}\right)<c} \mathfrak{p}_{i} \subseteq \bigcap_{\mathfrak{p} \in \operatorname{Ass}_{R}(R x)} \mathfrak{p}=\sqrt{\operatorname{Ann}_{R}(R x)} .
$$

So that there exists a positive integer $m$ such that $\left(\cap_{\mathrm{cd}\left(I, J, R / \mathfrak{p}_{i}\right)<c} \mathfrak{p}_{i}\right)^{m} x=0$. We claim that $x \in \cap_{\mathrm{cd}\left(I, J, R / \mathfrak{p}_{i}\right)=c} N_{i}$. Assume contrary that there is an integer $t$ such that $\operatorname{cd}\left(I, J, R / \mathfrak{p}_{t}\right)=c$ and $x \notin N_{t}$. Then by $\left(\cap_{\operatorname{cd}\left(I, J, R / \mathfrak{p}_{i}\right)<c} \mathfrak{p}_{i}\right)^{m} x=0 \in N_{t}$ and $x \notin N_{t}$ it follows that $\left(\cap_{\operatorname{cd}\left(I, J, R / \mathfrak{p}_{i}\right)<c} \mathfrak{p}_{i}\right)^{m} \subseteq \mathfrak{p}_{t}$ since $N_{t}$ is $\mathfrak{p}_{t}$-primary. Thus there exists some $\mathfrak{p}_{j}$ such that $\operatorname{cd}\left(I, J, R / \mathfrak{p}_{j}\right)<c$ and $\mathfrak{p}_{j} \subseteq \mathfrak{p}_{t}$. Hence, in view of Lemma $2.1, \operatorname{cd}\left(I, J, R / \mathfrak{p}_{t}\right) \leq \operatorname{cd}\left(I, J, R / \mathfrak{p}_{j}\right)<c$, which is a contradiction. So we have the desired result.

For the second part assume contrarily $H_{I, J}^{c}(N) \neq 0$, where $N=\cap_{\mathrm{cd}\left(I, J, R / \mathfrak{p}_{i}\right)=c} N_{i}$. Then $0:_{R} N=\cap_{i=1}^{n}\left(N_{i}:_{R} N\right)$ and so $\sqrt{0:_{R} N}=\cap_{\mathrm{cd}\left(I, J, R / \mathfrak{p}_{i}\right)<c} \sqrt{N_{i}:_{R} M}=$ $\cap_{\mathrm{cd}\left(I, J, R / \mathfrak{p}_{i}\right)<c} \mathfrak{p}_{i}$. Assume that $\mathfrak{p} \in \operatorname{Supp}(N)$, thus there is some $\mathfrak{p}_{j}$ with $\operatorname{cd}\left(I, J, R / \mathfrak{p}_{j}\right)$ $<c$ such that $\mathfrak{p}_{j} \subseteq \mathfrak{p}$. Hence, $\operatorname{cd}(I, J, R / \mathfrak{p}) \leq \operatorname{cd}\left(I, J, R / \mathfrak{p}_{j}\right)<c$. Then by the first paragraph of this section $\operatorname{cd}(I, J, N)<c$, which is a contradiction. Therefore, $H_{I, J}^{c}\left(N=\cap_{\mathrm{cd}\left(I, J, R / \mathfrak{p}_{i}\right)={ }_{c}} N_{i}\right)=0$.

Lemma 2.5. Let $R$ be a Noetherian local ring and $M$ be a non-zero finitely generated $R$-module of dimension $d$ such that $H_{I, J}^{d}(M) \neq 0$. Then there exists a positive integer $t$ such that $J^{t} M \subseteq T_{R}(I, J, M)$.

Proof. Let $0=\cap_{i=1}^{n} N_{i}$ denote a reduced primary decomposition of the zero submodule of $M$ where $N_{i}$ 's are $\mathfrak{p}_{i}$-primary submodules of $M$. By Theorem 2.4 we know that

$$
T_{R}(I, J, M)=\bigcap_{\operatorname{cd}\left(I, J, R / \mathfrak{p}_{i}\right)=d} N_{i}
$$

If $\operatorname{cd}\left(I, J, R / \mathfrak{p}_{i}\right)=d$, then $H_{I, J}^{d}\left(R / \mathfrak{p}_{i}\right) \neq 0$ and so by [4, Theorem 2.1], it follows that $J \subseteq \mathfrak{p}_{i}=\sqrt{\operatorname{Ann}_{R}\left(M / N_{i}\right)}$. Hence, there exists a positive integer $t_{i}$ such that $J^{t_{i}} M \subseteq N_{i}$. Set $t:=\max \left\{t_{i}: \operatorname{cd}\left(I, J, R / \mathfrak{p}_{i}\right)=d\right\}$. Then $J^{t} M \subseteq \cap_{\mathrm{cd}\left(I, J, R / \mathfrak{p}_{i}\right)=d} N_{i}$ and so we have the desired result by Theorem 2.4.

Corollary 2.6. Let $R$ be a Noetherian local ring, $I_{1}, I_{2}, J_{1}, J_{2}$ be ideals of $R$ and $M$ be a non-zero finitely generated $R$-module of dimension d. If $\operatorname{Att}_{R} H_{I_{1}, J_{1}}^{d}(M)=$ $\operatorname{Att}_{R} H_{I_{2}, J_{2}}^{d}(M)$, then the following statements are true:

(i) $T_{R}\left(I_{1}, J_{1}, M\right)=T_{R}\left(I_{2}, J_{2}, M\right)$.

(ii) There exist positive integers $t, s$ such that $H_{I_{1}, J_{1}}^{d}(M) \cong H_{I_{1}, J_{1}}^{d}\left(M / J_{2}^{t} M\right)$ and $H_{I_{2}, J_{2}}^{n}(M) \cong H_{I_{2}, J_{2}}^{n}\left(M / J_{1}^{s} M\right)$. 
Proof. (i) By assumption and [10, Theorem 3.1] for each $\mathfrak{p} \in \operatorname{Supp}_{R}(M)$ we have $\operatorname{cd}\left(I_{1}, J_{1}, R / \mathfrak{p}\right)=d$ if and only if $\operatorname{cd}\left(I_{2}, J_{2}, R / \mathfrak{p}\right)=d$. Now, in view of Theorem 2.4,

$$
T_{R}\left(I_{1}, J_{1}, M\right)=\cap_{\mathrm{cd}\left(I_{1}, J_{1}, R / \mathfrak{p}_{i}\right)=d} N_{i}=\cap_{\mathrm{cd}\left(I_{2}, J_{2}, R / \mathfrak{p}_{i}\right)=c} N_{i}=T_{R}\left(I_{2}, J_{2}, M\right),
$$

where $0=\cap_{i=1}^{n} N_{i}$ denotes a reduced primary decomposition of the zero submodule of $M$ and $N_{i}$ is a $\mathfrak{p}_{i}$-primary submodule of $M$.

(ii) In view of Lemma 2.5 and (i) we have $J_{2}^{t} M \subseteq T_{R}\left(I_{1}, J_{1}, M\right)$, for some positive integer $t$. Now by applying the functor $\Gamma_{I_{1}, J_{1}}(-)$ on the exact sequence

$$
0 \rightarrow J_{2}^{t} M \rightarrow M \rightarrow M / J_{2}^{t} M \rightarrow 0
$$

the desired result follows that is $H_{I_{1}, J_{1}}^{d}(M) \cong H_{I_{1}, J_{1}}^{d}\left(M / J_{2}^{t} M\right)$.

Theorem 2.7. Let $R$ be a Noetherian local ring and $M$ be a non-zero finitely generated $R$-module of dimension d such that $H_{I, J}^{d}(M) \neq 0$. Then $T_{R}\left(I, M / J^{t} M\right)=$ $T_{R}(I, J, M) / J^{t} M$, for some positive integer $t$.

Proof. By Lemma 2.5 there exists an integer $t \geq 1$ such that $J^{t} M \subseteq T_{R}(I, J, M)$. We show that $T_{R}\left(I, M / J^{t} M\right)=T_{R}(I, J, M) / J^{t} M$. Let $x+J^{t} M \in T_{R}\left(I, M / J^{t} M\right)$. Then $0=H_{I}^{d}\left(R\left(x+J^{t} M\right)\right) \cong H_{I, J^{t}}^{d}\left(R x / R x \cap J^{t} M\right)$ and so in view of [12, Proposition 1.4(8)], $H_{I, J}^{d}\left(R x / R x \cap J^{t} M\right)=0$. Also, as $R x \cap J^{t} M \subseteq T_{R}(I, J, M)$ it follows that $H_{I, J}^{d}\left(R x \cap J^{t} M\right)=0$. The exact sequence

$$
0 \rightarrow R x \cap J^{t} M \rightarrow R x \rightarrow \frac{R x}{R x \cap J^{t} M} \rightarrow 0
$$

induces an exact sequence

$$
\cdots \rightarrow H_{I, J}^{d}\left(R x \cap J^{t} M\right) \rightarrow H_{I, J}^{d}(R x) \rightarrow H_{I, J}^{d}\left(\frac{R x}{R x \cap J^{t} M}\right) \rightarrow 0 .
$$

Hence, it follows that $H_{I, J}^{d}(R x)=0$ and therefore, $x \in T_{R}(I, J, M)$. If $x \in$ $T_{R}(I, J, M)$, then $H_{I, J}^{d}(R x)=0$. Thus

$$
\begin{aligned}
H_{I, J}^{d}\left(R x / R x \cap J^{t} M\right) & \cong H_{I, J}^{d}\left(R\left(x+J^{t} M\right)\right) \\
& \cong H_{I, J^{t}}^{d}\left(R ( x + J ^ { t } M ) \cong H _ { I } ^ { d } \left(R\left(x+J^{t} M\right)=0\right.\right.
\end{aligned}
$$

by (*). Therefore, $x+J^{t} M \in T_{R}\left(I, M / J^{t} M\right)$.

Corollary 2.8. Let $R$ be a Noetherian local ring and $M$ be a non-zero finitely generated $R$-module of dimension d such that $H_{I, J}^{d}(M) \neq 0$ and let $J^{t} M \subseteq T_{R}(I, M)$ for some positive integer $t$. Then $T_{R}(I, J, M)=T_{R}(I, M)$.

Proof. By a similar argument to Theorem 2.7 one can show that $T_{R}\left(I, M / J^{t} M\right)=$ $T_{R}(I, M) / J^{t} M$ so that the result follows by Theorem 2.7 . 
Theorem 2.9. Let $R$ be a Noetherian local ring and $M$ be a non-zero finitely generated $R$-module of dimension $d$ such that $H_{I, J}^{d}(M) \neq 0$. Then the following statements are true:

(i) $\operatorname{Ann}_{R}\left(H_{I, J}^{d}(M)\right)=\operatorname{Ann}_{R}\left(M / T_{R}(I, J, M)\right)$.

(ii) If $J^{t} M \subseteq T_{R}(I, M)$ for some positive integer $t$, then $\operatorname{Ann}_{R}\left(H_{I}^{d}(M)\right)=$ $\operatorname{Ann}_{R}\left(H_{I, J}^{d}(M)\right)$.

Proof. (i) By Lemma 2.5 there exists a positive integer $t$ such that $J^{t} M \subseteq$ $T_{R}(I, J, M)$. Now, from the exact sequence

$$
0 \rightarrow J^{t} M \rightarrow M \rightarrow \frac{M}{J^{t} M} \rightarrow 0
$$

we have the exact sequence

$$
\cdots \rightarrow H_{I, J}^{d}\left(J^{t} M\right) \rightarrow H_{I, J}^{d}(M) \rightarrow H_{I, J}^{d}\left(\frac{M}{J^{t} M}\right) \rightarrow 0 .
$$

Since $J^{t} M \subseteq T_{R}(I, J, M)$, it follows that $H_{I, J}^{d}\left(J^{t} M\right)=0$ and so $H_{I, J}^{d}(M) \cong$ $H_{I, J}^{d}\left(M / J^{t} M\right)$. Thus $H_{I, J}^{d}(M) \cong H_{I, J^{t}}^{d}\left(M / J^{t} M\right) \cong H_{I}^{d}\left(M / J^{t} M\right)$. Hence, by $[1$, Theorem 2.3],

$$
\begin{aligned}
\operatorname{Ann}_{R}\left(H_{I, J}^{d}(M)\right) & =\operatorname{Ann}_{R}\left(H_{I}^{d}\left(M / J^{t} M\right)\right)=\operatorname{Ann}_{R}\left(\frac{M / J^{t} M}{T_{R}\left(I, M / J^{t} M\right)}\right) \\
& =\operatorname{Ann}_{R}\left(\frac{M / J^{t} M}{T_{R}(I, J, M) / J^{t} M}\right)=\operatorname{Ann}_{R}\left(M / T_{R}(I, J, M)\right) .
\end{aligned}
$$

(ii) The result follows by (i), Corollary 2.8 and [1, Theorem 2.3].

Corollary 2.10. Let $R$ be a Noetherian local ring with dimension $d$ such that $H_{I, J}^{d}(R) \neq 0$. Then $\operatorname{Ann}_{R}\left(H_{I, J}^{d}(R)\right)=T_{R}(I, J, R)$. So that $\operatorname{Ann}_{R}\left(H_{I, J}^{d}(R)\right)$ is the largest submodule of $R$ such that $\operatorname{cd}\left(I, J, \operatorname{Ann}_{R}\left(H_{I, J}^{d}(R)\right)\right)<d$.

Proof. It follows easily from Theorem 2.9(i) and Definition 2.2 .

Corollary 2.11. Let $R$ be a Noetherian local ring with dimension $d$ such that $H_{I, J}^{d}(R) \neq 0$. Then $\operatorname{Ann}_{R}\left(H_{I, J}^{d}(R)\right)=\cap_{\mathrm{cd}\left(I, J, R / \mathfrak{p}_{i}\right)=d} \mathfrak{q}_{i}$, where $0=\bigcap_{i=1}^{n} \mathfrak{q}_{i}$ is a reduced primary decomposition of the zero ideal of $R$ and $\mathfrak{q}_{i}$ is a $\mathfrak{p}_{i}$-primary ideal of $R$ for all $i$ with $1 \leq i \leq n$.

Corollary 2.12. Let $R$ be a Noetherian local ring of dimension $d \geq 1$ such that $H_{I, J}^{d}(R) \neq 0$. Then

$$
\operatorname{dim} R=\operatorname{dim} R / \operatorname{Ann}_{R}\left(H_{I, J}^{d}(R)\right)=\operatorname{dim} R / \Gamma_{I, J}(R) .
$$


Proof. The first equality follows by Corollary 2.10 and the second equality follows by $H_{I, J}^{d}(R) \cong H_{I, J}^{d}\left(R / \Gamma_{I, J}(R)\right)$, see $[12$, Corollary $1.13(4)]$. So $\Gamma_{I, J}(R) \subseteq$ $\operatorname{Ann}_{R}\left(H_{I, J}^{d}(R)\right)$.

Corollary 2.13. Let $(R, \mathfrak{m})$ be a Noetherian local ring and $M$ be a non-zero finitely generated $R$-module of dimension d such that $H_{I, J}^{d}(M) \neq 0$ and $T_{R}(I, J, M)=0$.

Then the following statements are true:

(i) $H_{I, J}^{d}(M) \cong H_{I}^{d}(M)$.

(ii) $H_{I, J}^{d}(R / \mathfrak{p}) \cong H_{I}^{d}(R / \mathfrak{p})$ for each $\mathfrak{p} \in \operatorname{Ass}_{R}(M)$ also $H_{I, J}^{d}(R / \mathfrak{p}) \cong H_{\mathfrak{m}}^{d}(R / \mathfrak{p})$ whenever $R$ is a complete ring.

(iii) $\operatorname{Att}_{R}\left(H_{I, J}^{d}(M)\right)=\operatorname{Att}_{R}\left(H_{\mathfrak{m}}^{d}(M)\right)=\operatorname{Assh}(M)$ and $\operatorname{Ann}_{R}\left(H_{I, J}^{d}(M)\right)=$ $\operatorname{Ann}_{R}\left(H_{\mathfrak{m}}^{d}(M)\right)$ whenever $R$ is a complete ring.

(iv) $\operatorname{Supp}_{R} M=V\left(\operatorname{Ann}_{R} H_{I, J}^{d}(M)\right)$.

Proof. (i) By assumption and Lemma 2.5 it follows that $J^{t} M=0$ for some positive integer $t$. Now the assertion follows by [12, Proposition 1.4(8)].

(ii) By assumption $\operatorname{cd}(I, J, R / \mathfrak{p})=d$ for all $\mathfrak{p} \in \operatorname{Ass}_{R}(M)$ thus $H_{I, J}^{d}(R / \mathfrak{p}) \neq$ 0 . On the other hand, if $\mathfrak{p} \in \operatorname{Ass}_{R}(M) \backslash V(J)$, then $\operatorname{dim} R /(\mathfrak{p}+J)<d$ and so $H_{I, J}^{d}(R / \mathfrak{p})=0$, by [12, Theorem 4.3]. Hence, for each $\mathfrak{p} \in \operatorname{Ass}_{R}(M)$ we have $J \subseteq \mathfrak{p}$ and therefore $R / \mathfrak{p}$ is a $J$-torsion $R$-module and so the first desired result. The second part follows by Lichtenbaum-Hartshorne Vanishing Theorem, see [3, Theorem 8.2.1].

(iii) The first part follows from (ii), [4, Theorem 2.1], [1, Corollary 3.4] and [3, Theorem 7.3.2]. By Corollary 2.3 we have $T_{R}(\mathfrak{m}, M)=T_{R}(I, M)=0$. Now the result follows from Theorem 2.9(i), [1, Corollary 2.7] and [2, Theorem 2.6].

(iv) It follows from Theorem 2.9(i).

Acknowledgement. The authors would like to thank the referee for the valuable suggestions and comments.

\section{References}

[1] A. Atazadeh, M. Sedghi and R. Naghipour, On the annihilators and attached primes of top local cohomology modules, Arch. Math., 102(3) (2014), 225-236.

[2] K. Bahmanpour, J. A'zami and Gh. Ghasemi, On the annihilators of local cohomology modules, J. Algebra, 363 (2012), 8-13.

[3] M. P. Brodmann and R. Y. Sharp, Local Cohomology: an Algebraic Introduction with Geometric Applications, Cambridge Studies in Advanced Mathematics, 60, Cambridge University Press, Cambridge, 1998. 
[4] L. Chu, Top local cohomology modules with respect to a pair of ideals, Proc. Amer. Math. Soc., 139(3) (2011), 777-782.

[5] L. Chu and W. Wang, Some results on local cohomology modules defined by a pair of ideals, J. Math. Kyoto Univ., 49 (2009), 193-200.

[6] C. Huneke and J. Koh, Cofiniteness and vanishing of local cohomology modules, Math. Proc. Cambridge Philos. Soc., 110(3) (1991), 421-429.

[7] L. R. Lynch, Annihilators of top local cohomology, Comm. Algebra, 40(2) (2012), 542-551.

[8] G. Lyubeznik, Finiteness properties of local cohomology modules (an application of D-modules to commutative algebra), Invent. Math., 113(1) (1993), $41-55$.

[9] L. T. Nhan and T. D. M. Chau, On the top local cohomology modules, J. Algebra 349 (2012), 342-352.

[10] Sh. Payrovi and S. Karimi, Upper bounds and attached primes of top local cohomology modules defined by a pair of ideals, J. Hyperstruct., 3(2) (2014), 101-107.

[11] P. Schenzel, Cohomological annihilators, Math. Proc. Cambridge Philos. Soc. 91(3) (1982), 345-350.

[12] R. Takahashi, Y. Yoshino and T. Yoshizawa, Local cohomology based on a nonclosed support defined by a pair of ideals, J. Pure Appl. Algebra, 213(4) (2009), 582-600.

\section{Susan Karimi}

Department of Mathematics

Faculty of Science

Payame Noor University

19395-3697 Tehran, Iran

e-mail: susan_karimi2@yahoo.com

Shiroyeh Payrovi (Corresponding Author)

Department of Mathematics

Imam Khomeini International University

34149-1-6818 Qazvin, Iran

e-mail: shpayrovi@sci.ikiu.ac.ir 